\title{
Linx
}

LinX Revue des linguistes de l'université Paris X Nanterre

$45 \mid 2001$

Invariants et variables dans les langues. Études typologiques

\section{La catégorie fonctionnelle du nom verbal en malgache (" classique ancien »)}

\section{Huguette Fugier}

\section{(2) OpenEdition}

\section{Journals}

Édition électronique

URL : http://journals.openedition.org/linx/828

DOI : $10.4000 / \operatorname{lin} x .828$

ISSN : 2118-9692

Éditeur

Presses universitaires de Paris Nanterre

Édition imprimée

Date de publication : 1 décembre 2001

Pagination : 127-138

ISSN : 0246-8743

Référence électronique

Huguette Fugier, «La catégorie fonctionnelle du nom verbal en malgache («classique ancien ») », Linx [En ligne], 45 | 2001, mis en ligne le 22 juin 2012, consulté le 06 mai 2019. URL : http:// journals.openedition.org/linx/828; DOI : 10.4000/linx.828

Ce document a été généré automatiquement le 6 mai 2019

Département de Sciences du langage, Université Paris Ouest 


\title{
La catégorie fonctionnelle du nom verbal en malgache (« classique ancien $»)$
}

\author{
Huguette Fugier
}

\section{Quel nom verbal, dans quel état de langue?}

1 1.1. Le Nom de procès en -ana. Comme l'ensemble des langues austronésiennes auquel il appartient, le malgache ne traite pas Nom et Verbe comme deux catégories fonctionnelles strictement séparées. La syntaxe malgache ménage en particulier, comme un pont entre les deux, une large place aux Noms verbaux. Parmi ces derniers -encore en attente d'une description générale- nous limiterons l'exposé d'aujourd'hui au Nom de procès dérivé sur thème verbal par le suffixe -ana.

Après l'avoir identifié (§ 2)

par différence avec les Noms verbaux dépourvus de suffixe (\$ 2.1)

et par sélection parmi les suffixés en -ana, en retenant de cet ensemble les seuls

Noms processifs à l'exclusion des statifs (\$2.2),

on pourra énumérer ses co-textes d'emploi, et inventorier par ce moyen les positions syntaxiques qu'il est capable d'occuper dans la structure de Phrase (§ 3).

3 1.2. En malgache « classique ancien » : les Tantara ny Andriana. Le corpus utilisé illustre l'état de langue dit: malgache "classique ancien » (DOMENICHINI-RAMIARAMANANA 1977 : 22). Il est prélevé sur l'immense recueil de documents relatifs à la monarchie merina, collectés par le P.F. CALLET sous le titre de Tantara ny Andriana, " Histoire des Rois ». De ces 1.217 pages j'ai examiné de plus près celles qui rapportent les didimpanjakana, les « lois du Royaume », édictées par Andrianampoinimerina avant 1810 (p. 756 sq. du vol. III de CALLET paru en 1878, réimprimé par l'Académie malgache en 1981). La raison de ce choix est double. D'une part la section de texte retenue présente de bonnes garanties d'authenticité, et par le même fait, d'homogénéité. Le P. CALLET semble en effet 
avoir obtenu les "lois", ainsi que les discours royaux ou délibérations associées, de notables Tsimahafotsy d'Ambohimanga, lesquels ayant vécu leur jeunesse dans l'entourage d'Andrianampoinimerina, en avaient mémorisé et en partie rédigé la teneur (DELIVRE 1974: 59-60). D'autre part, selon l'usage des rois d'Imerina, les «lois du Royaume » sont présentées au peuple sous forme d'un ou plusieurs discours (kabary), entre lesquels interviennent quelques délibérations avec les conseillers royaux ou dialogues avec tels représentants des populations : de sorte qu'un même contenu énoncé à plusieurs reprises non sans variations fournit autant de paraphrases spontanées bien instructives pour le linguiste.

N.B. Les textes cités, référenciés par exemple C. $800=$ CALLET p.800, seront donnés dans l'orthographe des Tantara. Le lecteur retrouvera facilement l'orthographe officielle actuelle au moyen de quelques correspondances simples, concernant :

La préposition suivie de l'article ny «le »:

CALLET aminy $N$ s'écrit aujourd'hui amin'ny $N$ « à propos du $N$ » mais atrehiny ny $N$ « « atrehin'ny $N$ « en présence du $\mathrm{N}$ »

Le N suivi du possessif postposé ny « de lui » :

CALLET vady ny s'écrit aujourd'hui vadiny « épouse de lui » isantoko ny « « isantokony « chaque région (de ce pays)"

Le $\mathrm{N}$ de procès suivi de l'article $n y$ « le » :

CALLET isaorany ny $N$ s'écrit aujourd'hui isaoran'ny $N$ «répudiation par le $N$ »

(complément d'agent)

mais fandevenany ny $N$ «

«fandevenana ny $N$ « le fait d'ensevelir les $N$ » (complément direct).

Sous chaque lexème ou morphème de la phrase citée figurera son sens lexical, et seulement s'il est besoin de la préciser, sa fonction syntaxique. En sus des sigles usuels : $\mathrm{N}$ = Nom, $\mathrm{V}=$ Verbe, $\mathrm{P}=$ Phrase, $\mathrm{c} . \mathrm{d} .=$ complément direct, l'abréviation crcl se lit: circonstanciel. La majuscule initiale d'un infinitif: Remercier, se lit: le procès de « remercier».

\section{Identification du nom de procès en -ana}

9 2.1. Noms verbaux avec ou sans suffixe : fanaovana vs fanao. Le malgache peut former sur un même radical verbal plusieurs Noms, distingués entre eux par leurs affixes. Le radical verbal comporte, outre le lexème $\mathrm{du} \mathrm{V}$, le ou les préverbe(s) qui accompagne(nt) ce dernier : 0 / -i- / -an- / -aha- / -amp-... Ce qui donne par exemple, une fois ajouté le morphème initial $m$-, signifiant du caractère verbal (ou plus précisément, signifiant de la forme verbale active),

Sur lexème tao « faire »: m-anao

Sur lexème teny « parler » : m-iteny.

10 Par commutation avec ce $m$-, le morphème $f$-formateur de Noms produit des Noms verbaux : ny fanao, ny fiteny. Or à ce fanao / fiteny s'oppose, par l'ajout d'un suffixe-ana, le $\mathrm{N}$ verbal dérivé fanaovana / fitenenana. Comme notre propos actuel n'inclut pas l'étude des emplois et valeurs du type fanao / fiteny, il suffira d'observer comment fanao et fanaovana se trouvent employés différentiellement dans une même $P$ des Tantara :

(1) C.800. Fa izao niandoha'ny ny fomba fanao aminy fikambanana

car / ceci / Commencer, $\mathrm{N}$ de procès passé / de eux / la / façon /faire / pour / les /

associations 
fanaovan'trano sy fanaovam-pasana sy fandevenany ny maty

construction / maisons / et / construction-tombeaux / et / ensevelissement / les /

morts

= «Car c'est à partir de là qu'ils adoptèrent la façon de faire quant aux associations pour la construction des maisons et la construction des tombeaux ainsi que pour l'ensevelissement des morts ».

11 On le voit : à l'expression nue du «faire » (fanao) répond l' « effectuation », le "faire » considéré dans les situations concrètes où l'on réalise effectivement maisons et tombeaux (fanaovana). Ce semble être une donnée générale de la dérivation que le $\mathrm{N}$ élargi de suffixes tend à évoquer le procès dans les circonstances (temps, moyens...) de sa réalisation : comme si, dans notre texte (1), ce qui s'exprime dans le cas de fanao par le moyen du lexème joint fomba résidait quand il s'agit de fanaovana dans le suffixe -ana. Cela dit fanaovana, même s'il est vrai que ce $\mathrm{N}$ dérivé facilite les interprétations circonstancielles, ne cesse pas pour autant d'être d'abord le nom du procès désigné par le lexème verbal qu'il inclut. La question intéressante devient alors de discerner ce qui le distingue du $\mathrm{N}$ verbal dépourvu de $f$ - désignant le même procès, à savoir : anaovana.

2.2. Noms verbaux avec ou sans préfixe : fisaorana vs isaorana. Fanaovana vs anaovana appartiennent à une série productive de paires liées par la relation $f$-vs non $f$-. Prenonsen pour exemple fisaorana vs isaorana tels que le texte (2) les distingue bien clairement. Ces deux $\mathrm{N}$ appariés reposent sur le radical saotra « remercier» et plus spécialement « répudier (une épouse)», présent dans le V actif misaotra ainsi que dans le passif voa saotra attesté ci-dessous :

(2) C. 792.

a) Raha anaty ny 12 andro ny isaorany ny lahy azy ka si / dans / les / 12 / jours / le Répudier, $\mathrm{N}$ de procès / (par) le / homme / elle / et vady tra-nenina na voa saotra aza dia vady ny hiany... épouse / regrette / même / répudiée / même / alors / épouse / de lui / de toute façon

Raha tsy manatrika indray ny isaorany ny lahy azy si / ne...pas / assiste à / une fois / le (fait de) Répudier / (par) le / homme / elle (sc.ny fokonolona) dia mbola vady ny hiany (sc. l'assemblée) / alors / encore / épouse / de lui / de toute façon

b) Ny aminy fisaoram-bady, izany koa ho lazai'ko aminareo le / à propos de / répudiation-épouse, N préfixé / ceci / aussi / (futur) / dit / par moi / à vous

ambaniandro sujets

= a) « Si la répudiation de elle par l'époux (a eu lieu) dans l'espace de 12 jours (sc. rétrospectivement) et que l'épouse est saisie de regret : alors elle est son épouse de toute façon... Si l'assemblée communale n'est pas témoin de la répudiation de elle par l'homme..., alors elle est encore son épouse de toute façon ».

b) = « En ce qui concerne la répudiation d'épouse, je vous dirai encore ceci, mes sujets ».

13 En position sujet du prédicat anaty ny 12 andro ou c.d. du V manatrika, et substantivé par l'article ny, isaorana en a) dénote le procès de "répudier », ou plus exactement une occurrence particulière de ce procès; ny fisaoram-bady en b) désigne au contraire la répudiation considérée comme une réalité en son état stabilisé, saisissable comme une notion qu'on peut citer et manipuler: de telle sorte que l'un est susceptible d'une valeur de vérité -Il a répudié: vrai ou faux ?- tandis que l'autre se prête particulièrement aux 
emplois résomptifs (MILNER 1982: 124). Quelques différences de fonctionnement les séparent d'ailleurs. Isaorana, ainsi qu'on vient de voir, accède aux fonctions actancielles, toutefois moins aisément que le $\mathrm{N}$ stabilisé par $f$ - En position gouvernée par une préposition, notamment, s'il est vrai que la forme passée nanomezana en (3) ne se laisse pas attribuer de façon sûre soit à anomezana soit à fanomezana, en revanche au temps présent notre corpus ne donne jamais que la forme en $f$-, exemplifiée par (4):

(3) C.806.Taminy nanomezan'angady ny lehilahy mifehy

lors de / Donner, $\mathrm{N}$ de procès passé / bêches / (à) les / hommes / exerçant autorité

$=$ « Lors de la remise des bêches aux chefs $»$.

(4) C.796. Ny aminy fanaovana trano

le / à propos de / construction, $\mathrm{N}$ verbal préfixé / maisons

$=\ll$ (Les prescriptions) relatives à la construction d'une maison $»$.

$\mathrm{Au}$ niveau syntagmatique non plus les propriétés communes ne masquent pas les divergences. D'un côté nos deux Noms admettent également une expansion nominale suffixée, laquelle vaut en tous les cas pour un génitif subjectif -de toutes façons dans une langue comme le malgache où les Noms verbaux sont transitifs, l' « objet » se réalise par un c.d.: et plus précisément, s'interprète comme génitif de l' «agent» et non du «possesseur »:

Ny (f)isaorana ny lahy azy

La / Répudiation, $\mathrm{N}$ de procès préfixable / (par) le / homme, « agent » / elle, c.d.

En revanche les démonstratifs, Adjectifs épithètes et expansions relatives, qui accompagnent fisaorana aussi naturellement qu'ils font de n'importe quel $\mathrm{N}$ prototypique, conviennent moins bien à la forme sans $f$-. Celle-ci n'exclut pas l'Adjectif : Ny isaorana azy tsy marina = «Le fait de la répudier, injuste »; du démonstratif le corpus fournit de rares exemples, ainsi C.813. Raha izao itondra'nao ny raza'nao izao = litt. " Si ce Veiller par toi (sur) tes ancêtres » c.à.d. « Si tu veilles ainsi sur tes ancêtres »; l'expansion relative semble impossible.

16 Mais pour isaorana l'essentiel n'est pas là. Moins typiquement nominal que fisaorana mais mieux approprié au rôle de prédicat sans fonctionner pourtant comme un $\mathrm{V}$, isaorana organise autour de lui des types de $\mathrm{P}$ originaux. Il intervient en effet en fonction prédicative

- \$3.1. en des positions dépendante

- \$ 3.2. dans l'une ou l'autre de deux P appariées par coordination ou corrélation

- \$3.3. dans une $P$ indépendante, en construction « circonstancielle ».

N.B. Pour alléger l'expression nous utiliserons désormais isaorana vs fisaorana pour symboliser par des termes concrets le $\mathrm{N}$ de procès vs le $\mathrm{N}$ stabilisé ; et de même misaotra (« remercier ») pour symboliser le V prototypique.

\section{Fonctions syntaxiques du nom de proces en -ana}

3.1. Isaorana en position dépendante. Par son côté prédicat, isaorana touche à misaotra : avec cette différence toutefois que, là où ce $\mathrm{V}$ réalisé à l'une quelconque des voix active / passive... dénote un procès qui s'accomplit effectivement, isaorana en tant que nom du procès se contente de désigner celui-ci c'est-à-dire de le mentionner sans asserter sa réalisation. Ce qui explique sa présence en diverses positions syntaxiquement dépendantes. 
19 3.1.1. Raha isaorana. $R a h a=$ « si, au cas où... " gouverne soit une $\mathrm{P}$ à deux constituants (sujet + prédicat) soit un constituant unique formant prédicat et Phrase à lui seul. Ce peut être soit un pronom personnel: Raha izahay Maintienindreny = "Quant à nous les Maintienindreny » (C.799); soit un N prototypique : Raha izao teny nao izao = "Quant à cette parole de toi » (ibid.) ; soit un $\mathrm{N}$ de procès comme isaorana précisément en :

(5) C.791. Ka raha isaorana tsy atrehiny ny fokonolona

et / si / Répudier, $\mathrm{N}$ de procès / ne...pas / en présence de / la / assemblée

$=$ « Et s'il y a répudiation non en présence de l'assemblée communale... ».

3.1.2. Tsapao ny tsena hizahana ... Le malgache dispose d'un morphème ho $/ h$ - à valeur lative "tourné vers l'avant ou l'avenir », pré-posable aux Verbes ou Adjectifs, voire aux Noms. Préfixé au V où il commute avec le $n$-de passé il reçoit une interprétation généralement future. Le $\mathrm{N}$ de procès admet aussi ce $h$ - latif ; c'est ainsi préfixé, et dans une interprétation plus intentionnelle que temporelle, qu'il occupe la position caractéristique de $\mathrm{P}$ seconde par rapport à une $\mathrm{P} 1$ précédente comportant un $\mathrm{V}$. Le lien entre les deux P implique la dominance syntaxique de P1 sur P2. Il consiste soit dans le izay subordonnant consécutif au V1 dans des expressions telles que mitady izay = «Chercher à faire que... »(C.774), tandremo izay = « Que l'on veille bien à ce que... » (C.782), atao izay = litt. «Il est fait que... » c.à.d. « On parvient à ce que... » (C.782), tsy hita izay = « On ne voit pas le moyen de faire que...» (C.807) ; soit dans le morphème latif préposé au prédicat de forme isaorana en $\mathrm{P} 2$, lequel latif n'étant pas tout à fait spécialisé comme morphème inscrit dans le système des temps semble jouer ici un rôle conjonctif. Les deux modes de liaison opèrent parfois ensemble dans la même $P$, soit qu'ils introduisent successivement deux prédicats secondaires coordonnés (Mitady ho " et » mitady izay en C.774), soit qu'ils agissent de façon simultanée sur le même prédicat secondaire (Mahalala izay hanaova'ko = « Décider que faire par moi » en C.779). Hizahana exemplifiera cet emploi $\mathrm{du} \mathrm{N}$ de procès futur en position de prédicat secondaire consécutif à une principale :

(6) C.807.Andeha tsapao ny tsena isantoko ny

allez ! / (que soient) inspectés,impér. passif / les / marchés / (de) chaque région / de lui

hizahana izay mora hanina

pour Voir, $\mathrm{N}$ de procès futur / lesquels / bon marché / nourriture

= «Allez, que soient inspectés les marchés de chaque région (du pays), pour voir lesquels ont des vivres bon marché ».

21 L'affinité de isaorana avec le latif de futur intentionnel n'étonnera personne : quoi de plus naturel que d'affecter à l'événement / état (seulement) visé le $\mathrm{N}$ qui désigne le procès sans prendre parti sur sa réalité ?

Dans la même position, l'interprétation consécutive n'est pas moins naturelle quand il s'agit de décrire le comportement d'un agent opérant « de telle sorte que » tel événement / état s'ensuive de façon prévisible. De fait cette interprétation s'attache souvent au $\mathrm{N}$ de procès: avec, voire sans le $f$ - préfixal, parfois appuyé par la particule assertive et légèrement optative $m b a$ :

(7) C.757. maty nay atosy kay, tsy mba itiava'

tué / par nous / repoussé / par nous / ne...pas / (confirmatif) / Aimer, $\mathrm{N}$ de procès nay havana

par nous / parents 
= (Si quelqu'un viole les lois d'Andrianampoinimerina) «il est mis à mort par nous, exclu par nous, de sorte qu'il n'y ait pas d'affection pour nos parents » c.à.d. "sans prise en compte des affections familiales ».

Cf. C.759. Dia maty nay ambanilanitra, fa tsy mba iandrasa'nay anao = «Ils seront mis à mort par nous tes sujets, et sans t'attendre »;

Et C.787. Fa tsy anareo ny tany fa ahy, hoy Andrianampoinimerina, ihinana'nareo ny vokatra = "Car la terre n'est pas à vous mais à moi, dit Andrianampoinimerina, de sorte que vous consommiez le produit » c.à.d. « avec jouissance par vous du produit ».

Cette valeur de conséquence ou modalité du procès signifié par V1 aboutit à un emploi quasi-adverbial de la forme isaorana, par exemple: Manao tetika iraisana « Ils montent un complot en se mettant d'accord " c.à.d. " ensemble» (C.763) ; Nampitari-bato ny vahoaka niandohany = «Il fit charrier des pierres à la population en commençant » c.à.d. «pour commencer, tout d'abord » (C.800).

3.1.3. Tsy mety ifonany ny fokonolona izy. Dans l'ensemble de la syntaxe malgache plusieurs V impersonnels dont mety «il est possible», tsy azo "il n'est pas admis» gouvernent normalement la forme isaorana: toute autre construction se heurterait en effet à l'incapacité de ces impersonnels à gouverner un $\mathrm{V}$ actif / passif...-incapacité tenant elle-même à un problème de sujet (question de structure traitée dans FUGIER 1999: 67). Cependant mety connaît aussi un emploi personnel et dans ce dernier, en P négative c'est-à-dire en valeur " ne pas consentir à... », il se trouve dans notre Tantara gouverner aussi un $\mathrm{N}$ de procès :

(8) C.781.Ary raha tsy mety ifonany ny fokonolona izy

et / si / ne...pas / acceptent / S'excuser, $\mathrm{N}$ de procès / la / assemblée / ils

$=$ «Et s'ils (sc. les propriétaires lésés) n'acceptent pas la démarche d'excuse de l'assemblée communale...».

Pareille situation suggère qu'à la raison de structure syntaxique se superpose apparemment une autre plus générale, c'est-à-dire commune aux emplois personnels et impersonnels et jouant surtout dans le champ d'une négation : raison qui tient cette fois à la valeur modale des expressions tsy mety, tsy azo : tant il est vrai qu'au procès présenté en dépendance syntaxique de ces expressions c'est-à-dire sous la modalité du « non possible, non admis ", la simple mention du procès convient mieux, s'insère mieux dans la visée communicative du locuteur, que l'assertion véhiculée par le $\mathrm{V}$, selon laquelle «ce procès s'est vraiment effectué ». De façon générale et sous réserve d'une vérification qui examinerait soigneusement les co-textes et comptabiliserait exemples et contreexemples, une compatibilité particulière semble associer les $\mathrm{N}$ de procès aux co-textes négatifs, comme en témoignent les séquences courantes de forme ny tsy iavonany ny olona raha mitari-bato $=\ll$ Le fait que les gens ne montrent pas d'arrogance quand ils charrient des pierres » (C.799); ny tsy ihoaram-bava aman'olona $=$ «Le fait de ne pas se livrer à des excès verbaux envers les gens » (C.799); ou, dans le champ de raha tsy : Raha tsy andoavana volatsivaky $=$ «Si l'on ne paie pas une piastre entière » (C.787).

Son statut de $\mathrm{N}$ de procès n'agit pourtant pas comme une contrainte qui empêcherait isaorana d'intervenir aussi en position non gouvernée. Il n'est pas vrai non plus qu'aucun site d'accueil n'existe pour isaorana hors de l'alternative: position gouvernée vs indépendante: à preuve sa présence régulière et signifiante dans les paires de Phrases syntaxiquement équipollentes. 


\subsection{Isaorana, prédicat : \\ d'une $\mathrm{P}$ seconde dans une paire de $\mathrm{P}$ coordonnées d'une $P$ première dans une paire de $P$ corrélées}

27 Il arrive que deux Phrases, chacune syntaxiquement indépendante, forment ensemble une paire, liée ou non par un morphème explicite, unifiée en tous cas par un contour intonationnel qui en assure la cohérence. Or de ces deux $\mathrm{P}$, l'une antérieure et l'autre postérieure en ordre linéaire -nommons-les par convention $\mathrm{Pa}$ et $\mathrm{Pb}-$, une seule réalise son prédicat par la forme isaorana: ceci, en une distribution non quelconque puisqu' isaorana selon qu'il intervient en $\mathrm{Pb}$ ou $\mathrm{Pa}$ signale entre les deux $\mathrm{P}$ une relation soit de coordination soit de corrélation.

\subsection{1. $\mathrm{V}+$ isaorana : une coordination. Le dispositif}

$$
\begin{aligned}
& \mathrm{Pa}+\mathrm{Pb} \\
& \mathrm{V}+\text { isaorana }
\end{aligned}
$$

réalise une séquence de coordination. Liés par un connecteur cumulatif « et, mais », les deux termes y font l'objet de propositions successives, chacune posée et vérifiée indépendamment de l'autre. Parmi les connecteurs $k a$ «et (avec une légère valeur consécutive) » l'emporte de beaucoup par le nombre d'occurrences :

(9) C.760. (Fa raha manao io hianareo) andrao mikoro car / si : faites / cela / vous / il est à craindre (que) / faire du désordre ka tsy ahazoa' ko ny fanjakana

et / ne...pas / Posséder, $\mathrm{N}$ de procès / par moi / le / royaume

$=\ll($ Car si vous faites cela $: \mathrm{sc}$. vous adonner à la boisson), il est à craindre que le désordre n'apparaisse et que je ne tienne plus en main le royaume».

Mais se trouvent aussi ary « et » (C.807), dia « et puis » (C.807), kanjo « cependant » (C.786). Si la série coordonnée comporte plus de deux termes, la forme isaorana affecte le dernier, comme en C.807: hahazina $(=\mathrm{V})+$ hahabetsaka $(=\mathrm{V})+$ handehandehanany ny olona $(=\mathrm{N}$ de procès avec son agent suffixé). Les rares cas de figure qui s'écartent de ce schéma ne seront pas vraiment qualifiés de déviants :

- Soit que d'une série comportant trois termes, le second emploie isaorana tandis que le dernier revient à la forme du V actif / passif... comme si le discours rebondissait là pour un nouveau départ : par exemple dans C.777, où

\begin{tabular}{|l|l|l|l|l|}
\hline 1. Hiady & + & 2 . anaovana & + & 3.ome-nao \\
\hline V actif & + & N de procès & + & V passif \\
\hline
\end{tabular}

- Soit que Pa se trouve gouverné par la conjonction raha «si », sans changement pour autant de la relation $\mathrm{V}$ vs isaorana, comme en :

(10) C.801. Ary raha may ny trano 5 no miakatra :

et / si / brûlent, Adj. préd. / les / maisons / 5 / et / au-dessus

dia amonoana omby

alors / Tuer, $\mathrm{N}$ de procès / boeuf

$=$ « Et si prennent feu 5 maisons et plus : Sacrifier un bœuf $»$. 

.796. Auquel cas la séquence ainsi réalisée Raha $V$, dia isaorana montre un léger empiètement du schéma coordonnant sur le domaine de la subordination : «Si Pa, alors $\mathrm{Pb}$ » étant traité comme « $\mathrm{Pa}$ est supposé admis, et alors $\mathrm{Pb}$ ».

3.2.2. Isaorana $+\mathrm{V}$ : une corrélation. Entre les deux $\mathrm{P}$ indépendantes successives peut exister aussi un lien de corrélation. La corrélation consiste en ceci, que la $\mathrm{Pb}$ n'est formulée qu'en relation avec la Pa préalablement posée. Pour exprimer ce rapport ordonné c'est-à-dire non susceptible d'interversion-le malgache dispose de plusieurs procédés, qui font intervenir :

- soit un relateur tel que no, capable d'ordonner $\mathrm{Pb}$ vs $\mathrm{Pa}$, selon le schéma (non approfondi aujourd'hui) : (sujet $+\mathrm{Va}$ ) no (sujet $+\mathrm{Vb}$ )

- soit précisément le $\mathrm{N}$ de procès isaorana formant à lui seul la $\mathrm{Pa}$.

Deux propriétés caractérisent respectivement la $\mathrm{Pa}$ entière, et isaorana en particulier. D'une part cette $\mathrm{Pa}$, telle qu'énoncée par un locuteur malgachophone, présente une montée de la voix vers l'aigu juste avant la pause séparant (avec ou sans relateur) les deux $P$ l'une de l'autre: comme en une fin d'énoncé interrogatif et dans l'attente de la suite. D'autre part isaorana admet, voire sollicite ici une interprétation de «circonstance »-que le décodeur sélectionnera à partir du contexte parmi les circonstances de «manière / cause... » également envisageables. La relation des contenus rapportés en $\mathrm{Pa}$ et $\mathrm{Pb}$ est la suivante. $\mathrm{La} \mathrm{Pa}$ énonce un événement (une situation) déjà connu du destinataire ou considéré comme admis : mais que sa formulation par un $\mathrm{N}$ verbal dérivé en -ana suggère de considérer dans son lieu de survenance / sa manière / sa cause... : lequel lieu... sera précisément donné à connaître par la $\mathrm{Pb}$ subséquente, sous forme (sujet $+\mathrm{V}$ ) et en intonation assertive. Le lecteur francophone comprendra sans peine ce dispositif puisque dans sa langue aussi le $\mathrm{N}$ construit sur radical verbal s'interprète en contexte idoine comme « lieu / manière / cause... ». Qu'il imagine donc un N comme :

Rencontre interprété « lieu où l'on se rencontre ", dans une séquence de forme Leur rencontre : ils s'étaient donné rendez-vous dans une cabane de berger ;

Présentation interprété "manière dont on présente, circonstances dans lesquelles... », dans La présentation du prototype : la presse spécialisée sera convoquée ;

Explosion interprété "cause pour laquelle on explose", dans L'explosion révolutionnaire : les paysans ne supportaient plus la corvée.

Le dispositif malgache : (Ny) isaorany ny vady: dia $\mathrm{Pb}=$ «Le Répudier par lui l'épouse : eh bien il y avait tel événement, telle situation ", ce dispositif ne fait rien d'autre que régulariser dans la langue ce qui reste en français à la limite de l'usage. Le fait que isaorana se présente, soit pourvu, soit dépourvu d'article, qui change quelque peu la structure syntagmatique du $\mathrm{N}$ de procès constituant $\mathrm{Pa}$, ne modifie pas la relation de $\mathrm{Pb}$ à $\mathrm{Pa}$. Les quelques exemples ci-dessous ne se distinguent donc que par l'interprétation :

En (11) « manière, circonstances du procès », telles que $\mathrm{Pb}$ les explicitera

En (12) « circonstances concomitantes »

En (13) « causes (déterminantes et finales) ».

(11) Formule par laquelle le manuscrit $A$ résume les règnes des premiers rois d'Imerina, citée par DELIVRE : 55.

Ny nanjakan-dR nanambady an-dR ...

Le / Régner, $\mathrm{N}$ de procès passé / (de) R. / épousa, $\mathrm{V}$ actif passé / R. niteraka an-dR

engendra, $\mathrm{V}$ actif passé / $\mathrm{R}$.

$=$ « Le règne de $\mathrm{R}$ : il épousa $\mathrm{R}$, eut pour fils $\mathrm{R}$ ». 
(12) C.756. Ny namoahan' Andrianampoinimerina ny

le / Publier, $\mathrm{N}$ de procès passé / (par) Andria..., $\mathrm{N}$ propre / les

didy, dia namory ny ambanilanitra Andrianampoinimerina

lois / eh bien / rassembla, V actif passé / les / sujets / Andria..., N propre

teto Antananarivo

là / (à) Antana..., N propre

= litt. « La publication par A. des lois : eh bien, A. rassembla ses sujets à Antananarivo ».

(13) C.804. Anomeza'ko ity vola ity : faly sy ravo

Donner, $\mathrm{N}$ de procès / par moi / ce / argent / ce / content / et / joyeux

aho. Anomeza' ko anareo iny vola iny : enti' nareo

je / Donner / par moi / à vous / ce / argent / ce / utilisé / par vous

manantatra ny vary nareo

goûter en prémices / le / riz / de / vous

= litt. « Le don par moi (de) cet argent : je suis content et joyeux... Le don par moi (de) cet argent : il est utilisé par vous à goûter votre riz ».

Comme on le voit, entre les deux énoncés $(\mathrm{V}+$ isaorana) et (isaorana $+\mathrm{V})$ ce qui diffère est à la fois l'ordre linéaire et le rôle joué par isaorana dans l'organisation de l'énoncé. En deuxième terme d'une séquence coordonnée le $\mathrm{N}$ de procès n'est rien d'autre qu'un phénomène d'économie puisqu'effectivement, des deux $\mathrm{P}$ successives il suffit que la première présente une fois pour toutes un $\mathrm{V}$ personnel pour que les propriétés verbales dont la propriété sémantique d'asserter la réalité du contenu énoncé- bénéficient à la paire entière. En premier terme d'une paire corrélée isaorana sert pareillement à des fins d'économie puisqu'il ramasse à la façon d'un $\mathrm{N}$ résomptif l'information déjà connue, mais cette économie entre dans une stratégie différente. Car il s'agit ici de laisser $\mathrm{Pb}$ développer par un $\mathrm{V}$ personnel l'information nouvelle et attendue (concernant la «manière / cause...» du procès $\mathrm{Pa}$ ) ; et d'établir ainsi entre les deux $\mathrm{P}$ une relation dissymétrique quoique non subordonnante. Un tel système d'association inégale entre deux $P$ équipollentes existe en maintes langues, d'une façon telle que la dissymétrie réalisée en malgache par isaorana vs misaotra s'exprime souvent ailleurs par un jeu de corrélateurs externes, comme le cum...tum des langues indo-européennes (HAUDRY 1973; dans les langues indiennes modernes, cf. MONTAUT 1999 : 74-78).

3.3. Isaorana prédicat d'une Phrase indépendante: la construction " circonstancielle ". La construction bien décrite dans les grammaires sous le nom de voix circonstancielle repose sur la relation entre une forme isaorana et un constituant réalisé dans la grande majorité des cas par un $\mathrm{N}$ prototypique. Or ce qui fait problème n’est pas tant de décrire les deux termes (\$ 3.3.1) que de bien saisir leur relation (§ 3.3.2).

3.3.1. Le $\mathrm{N}$ verbal dérivé en -ana dénote un procès, plus particulièrement regardé dans ses circonstances, c'est-à-dire en tant qu'il se produit « à tel propos / de telle manière... ». Quant au N co-présent il désigne justement la circonstance actuellement considérée, qui va faire sens avec isaorana. Cette circonstance sera, dans notre corpus en particulier :

le quelque chose «à propos de quoi il y a procès de... ( (sens général) comme dans l'exemple (14)

le quelque chose « selon, conformément à quoi... » (sens de « manière » comme dans l'exemple (15)

le quelque chose "depuis, à partir de quoi... » (sens d' "origine ») comme dans l'exemple (16)

le quelque chose «en raison de quoi... " (sens de «cause justifiante ») comme dans l'exemple (17).

(14) C.815. F' aza ny ai'nareo atahora'nareo 
mais / que...ne..pas / la / vie / de vous / Craindre, $\mathrm{N}$ de procès / par vous fa ny fanjaka'ko

mais / le / royaume / de moi

= litt. «Que mon royaume et non point votre vie soit à propos de quoi vous éprouvez de la crainte ».

(15) C.798. Raha izao kosa, Andrianampoinimerina, no itondra'

si / ceci / cependant / Andria..., $\mathrm{N}$ propre / (connecteur) / Gouverner, $\mathrm{N}$ de procès nao anay

par toi / nous, c.d.

= litt. « Si ceci est, Andrianampoinimerina, la façon dont vous nous gouvernez... ».

(16) C.803. Andrianampoinimerina nisehoany ny « namoaka angady »

Andria..., $\mathrm{N}$ propre / Apparaître, $\mathrm{N}$ de procès passé / le / attribuer, V actif passé /

bêches

= litt. «Andrianampoinimerina est celui à partir de qui apparut la distribution des bêches".

(17) C.795. K' izany no anaovana didy hoe...

et / ceci / (connecteur) / Faire, $\mathrm{N}$ de procès / loi / (introducteur de discours)

$=\ll$ (La loi du mariage est préjudiciable au pays), et ceci est la raison pour laquelle on fait une loi qui dit... ».

3.3.2. La doctrine usuelle issue des travaux fondateurs de J. WEBBER et D. GRIFFITHS au milieu du XIX ${ }^{\circ}$ siècle analyse isaorana comme une voix verbale au même titre que le passif, et le $\mathrm{N}$ co-présent, comme le sujet de ce $\mathrm{V}$ à la voix circonstancielle. Cependant trois données de fait objectent à cette interprétation.

39 a) Le constituant associable à isaorana ne subit pas les mêmes contraintes de sélection que le sujet d'un V: ce dernier est beaucoup plus contraint. Car on trouve associé à isaorana, dans notre corpus :

40 - Soit un constituant déjà situé syntaxiquement dans le champ de dépendance de isaorana, donc indisponible pour assurer en même temps la fonction incompatible de sujet. Ce pourrait être le cas en (18) s'il est vrai que izao représente ici l'objet interne du N de procès ananarana (radical anatra), valant sémantiquement pour le contenu des « conseils » dispensés aux orphelins :

(18) C.805. Izao nananaran'Andrianampoinimerina ny kamboty ceci / Conseiller, $\mathrm{N}$ de procès passé / Andria..., $\mathrm{N}$ propre / les / orphelins $=«$ En ceci consistaient les conseils d'Andrianampoinimerina aux orphelins $»$.

41 - Soit, à l'inverse, un constituant syntaxiquement extérieur à la $\mathrm{P}$ comme ahoana « pourquoi ? » en (19), dans ce dialogue entre un conseiller royal partisan de la clémence en matière pénale, et le roi :

(19) C.773. Ahoana no ameloma'nao azy?

pourquoi / (connecteur) / Faire vivre, $\mathrm{N}$ de procès / par toi / lui, c.d.

$=$ « (- Il y a des condamnés qui allaient être exécutés et que j’ai fait vivre) .- Pourquoi donc les as-tu fait vivre?».

42 Ainsi compatible avec isaorana, ahoana accéderait-il à la position de sujet auprès d'un V sous quelque voix que celui-ci se présente? S'il apparaît bien que non, alors le champ de la compatibilité avec isaorana déborde celui accordé à la fonction subjectale.

b) Isaorana reste en continuité formelle avec le $\mathrm{N}$ verbal : il lui suffit de s'adjoindre le morphème $f$ - signifiant le caractère habituel du procès pour coïncider avec le $\mathrm{N}$ stabilisé fisaorana « remerciement ». Il est bien entendu que ce $f$ - se prête aussi à d'autres formes, 
adjectivales ou verbales (fahita, fanao). Mais une forme comme fakana " procès habituel de prendre » s'avère vraiment très proche de ny fakana « la prise " sans cesser pour autant de fonctionner comme akana dans un énoncé comme celui-ci, à propos des rites de circoncision :

(20) C.788. Izao no ranomasina alaina, sy Andranomiry

ceci / (connecteur) / eau sainte / cherchée, passif / et / Andrano..., N propre

fakana ranomahery kosa Andranomiry

prendre, $\mathrm{N}$ verbal préfixé / eau puissante / (connecteur) / Andrano..., N propre

$=$ «Telle est l'eau sainte qu'on va chercher, et c'est à Andranomiry qu'on prend (sc. toujours, dans la continuité de la tradition) l'eau puissante -à Andranomiry ».

c) De la construction « circonstancielle » à la construction subjectale l'ordre considéré comme non marqué qui règle la succession linéaire des deux constituants majeurs diffère polairement puisque dans une $\mathrm{P}$ (misaotra avec son sujet), sauf raison d'expressivité le $\mathrm{V}$ précède le sujet tandis que isaorana suit normalement le terme de « circonstance » qui lui est associé. La place initiale soulignée par no c'est-à-dire emphatisée accordée à la "circonstance» n'est ni quelconque ni aléatoire: tout l'équilibre de la construction « circonstancielle » repose sur ce rapport entre un terme emphatisé et le $\mathrm{N}$ de procès. Le locuteur ne déroge à cet ordre que par l'effet d'exigences co-textuelles, par exemple en C.768 le $\mathrm{N}$ de «circonstance " ireo didy suit son $\mathrm{N}$ de procès namolahana (radical folaka " dompter, amener à obéir ») parce que le $\mathrm{N}$ désignant « ces lois ", maintes fois énoncé en amont, n'a plus besoin d'être emphatisé ; tandis qu'à l'inverse en C.784 itomaniana (radical tomany « pleurer ») précède le $\mathrm{N}$ alina " nuit » pour produire une série bien rythmée avec la séquence passive antérieure alevina alina $=$ "(Le roi) est enterré de nuit ». Mais si l'ordre ( $\mathrm{N}$ de « circonstance » + isaorana) reste effectivement la règle, c'est bien que par ce choix d'organisation linéaire, la construction « circonstancielle " situe isaorana à l'opposé de l'ensemble formé par les voix verbales.

Plusieurs données opposent donc le isaorana de la construction «circonstancielle » aux voix du $\mathrm{V}$ malgache, et confirment son lien maintenu avec le $\mathrm{N}$ verbal. Ce $\mathrm{N}$ dérivé en -ana soutient tout un ensemble de constructions dans la syntaxe malgache. Bien à sa place dans les positions dépendantes, outil économique de la coordination, il forme par assemblage dissymétrique avec une $\mathrm{P}$ assertive une structure originale de corrélation; et le même procédé associant le $\mathrm{N}$ du procès (seulement) nommé à un autre constituant sinon forcément nominal, du moins capable d'exprimer une modalité ou circonstance du procès- se trouve encore au fondement de la construction « circonstancielle ».

Voir dans isaorana un $\mathrm{N}$ verbal plutôt qu'une voix ne revient pas à lui dénier toute relation avec le système des voix malgaches. Tout au contraire! Isaorana se coordonne avec n'importe quelle voix verbale. Surtout, il supplée éventuellement l'une quelconque des voix secondaires, c'est-à-dire que toute $\mathrm{P}$ constituée par: sujet «destinataire / bénéficiaire" + voix destinative / bénéfactive, ou par: sujet "instrument» + voix instrumentive, se remplace aisément dans le même co-texte par: $\mathrm{N}$ de « circonstance » (= " destinataire / bénéficiaire / instrument ») + isaorana. Les descriptions fournies par des ouvrages classiques gardent naturellement ici toute leur pertinence (RAHAJARIZAFY $1960: 27$; RAJAONA $1972: 128$ ). Mais c'est justement parce que isaorana n'appartient pas à l'ensemble de ces voix secondaires qu'il se prête à suppléer n'importe laquelle d'entre elles.

On le voit, isaorana s'intègre parfaitement au fonctionnement de la syntaxe malgache. Qu'il serve à organiser dans une $\mathrm{P}$ l'information déjà connue ou secondaire vs 
l'information saillante emphatisée, et qu'on puisse le décrire en tant que tel, n'empêche que ce $\mathrm{N}$ de procès trouve sa place dans des contextes syntaxiques précis, sert d'instrument à la coordination et surtout à la corrélation, et crée un type de $\mathrm{P}$ régulièrement inscrit dans la grammaire. C'est ainsi une originalité de la langue malgache que de fonder une partie non négligeable de sa syntaxe sur cette classe morphosyntaxique très particulière qu'est le $\mathrm{N}$ de procès.

\section{BIBLIOGRAPHIE}

DELIVRE, Alain (1974) L'Histoire des Rois d'Imerina. Interprétation d'une tradition orale, Klincksieck, Paris.

DOMENICHINI-RAMIARAMANANA, Bakoly (1977) Le malgache. Essai de description sommaire, SELAF, Paris. FUGIER, Huguette (1999) Syntaxe malgache, Peeters, Louvain-la-Neuve.

HAUDRY, Jean (1973) «Parataxe, hypotaxe et corrélation dans la phrase latine », in Bulletin de la Société de Linguistique de Paris 68 / 1 :147-186.

MILNER, Jean-Claude (1982) «De l'existence du sujet dans les groupes nominaux », in Ordres et raisons de la langue, éd. Milner J.C., Seuil, Paris : 123-139.

MONTAUT, Annie (1999) «La corrélative et le quotatif dans les langues indiennes : deux systèmes qu'on a intérêt à distinguer de la subordination ", in Typologie des langues, Universaux linguistiques, éd. Sörés, A. et Marchello-Nizia, C., nº spécial de LINX : 73-88.

RAHAJARIZAFY, Antoine (1960) Essai sur la grammaire malgache, Antanimena, Antananarivo.

RAJAONA, Siméon (1972) Structure du malgache, Ambozontany, Fianarantsoa.

Corpus

CALLET, François (1878) Tantara ny Andriana, t.III, réimpr. par l'Académie malgache, 1981.

\section{AUTEUR}

HUGUETTE FUGIER 